\title{
Current E-education Problems in China - Misgivings about PPT Courseware Production
}

\author{
Chi Zhang \\ Foreign Language Department, Hubei University of Automotive Technology, Shiyan, Hubei, China (Zhangc58@ 126.com)
}

\begin{abstract}
In recent years, there has been a rapid development of e-education in China as well as a vast increase of electronic resources. However, the quality of some electronic resources is worrisome. This paper describes the deficiencies of certain PPT courseware using the structure analysis of certain English textbooks and the matching courseware, demonstrates two major categories of paragraph structure analysis error in the existing courseware, explores the problems of e-education, analyzes some root causes of these problems, at last comes to a conclusion and makes some further recommendations.
\end{abstract}

Index Terms - e-education, electronic resources, PPT courseware, text structure analysis, deduction, induction

\section{Introduction}

Since the Chinese economic reform, China's college English education has been improving substantially, but it still cannot meet the needs of China's socio-economic development. December 2002, the higher education department of Ministry of Education officially launched the "College English Teaching Revision" project, and the College English Teaching Revision became a focal point to the Ministry of Education, and is included in the "Higher Education Reform and Teaching Quality Improvement Project". After over ten years of effort, college English teaching has made many great achievements, such as, the establishment of College English Curriculum Requirements (For Trial Implementation), the publication of numerous papers, monographs, and English textbooks, the research and development of some electronic resources, the exploration of various teaching theories, methods and tools, as well as the construction of college English teaching model based on computers and networks. Currently, most universities have adopted the multimedia teaching method, namely by means of computers, and the support materials of PPT and CD-ROM resources for teaching. However, due to constraints of manpower, material resources and time factors, some multimedia content lacks scientific backing and structure, oftentimes inconsistent with textbooks. This often causes confusion and difficulties in the teaching process. This article uses the Structure Analysis of New Horizon College English Reading and Writing (Second Edition) and their matching PPT courseware in attempts to explore the problems of e-education, their root causes, and tries to make some recommendations.

\section{Confusion of the Text Structure Analysis}

In Book One of New Horizon College English Reading and Writing (Second Edition), eight paragraph patterns or structures are introduced in the Text Structure Analysis parts in each unit. They are "a paragraph of cause and effect, a paragraph of sequenced order of events, a paragraph of a general point supported by examples, a paragraph of a statement supported by a list of examples, a paragraph of a general statement supported by a problem-solution pattern, a paragraph of a set of sequential actions, paragraphs of a topic supported by details, and a paragraph of a general principle supported by an example." ${ }^{[1]}$ In Book Two, Book Three and Book Four, ten more paragraph patterns or structures are introduced (not including repeated analyses). I don't want to talk about whether it is appropriate to use so much space analyzing paragraph patterns or structures and I don't want to talk about whether this kind of arrangement and classification is reasonable or scientific. What I do want to talk about is the matching PPT courseware made by Air Force University of Engineering and Foreign Language Teaching \& Research Press, where there are more serious problems.

To begin with, the mentioned PPT courseware introduces a lot of devices for developing paragraphs, such as "paraphrases of the two key words, narration, sequence of time and space (topic_cause- effect), description of sequential actions ( topic —action 1-action 2-...), exemplification, listing (general statement —a list of examples), questioning (set a scene-question 1 -question 2-...), phenomenon description, deduction, induction, induction through introspection, ${ }^{[2]}$ to name a few. Are there any differences between these devices and paragraph structures or paragraph patterns? It seems that some do and some don't. Then, why so many terms, so many confused classifications? Don't they make things more complicated? Moreover, some of the terms or devices are untenable arguments and some are fallacies, such as the "deduction" structure analysis and the "induction" structure analysis. In the following two sections, these two fallacies in the PPT courseware are refuted respectively.

\subsection{Deduction or a statement supported by reasons?}

In the Text Structure Analysis parts of this PPT courseware, some texts' or paragraphs' developing devices are analyzed as "deduction". For example, in Unit 9, Book One, Paragraph 4 of Section A is analyzed as following:

"Para. 4 is developed through deductive reasoning:

The first tip is: don't get behind (Premise). The problem of studying, hard enough to start with, becomes almost impossible when you are trying to do three weeks' work in one weekend. Even the fastest readers have trouble doing that. And if you are behind in written work that must be turned in, the teacher who accepts it late will probably not give you full credit. Perhaps he may not accept it at all. Most teachers believe that it is your responsibility to do work according to a 
reasonable plan, and they expect you to take it seriously (Deductive reasoning). Little room is given to students that are not able to manage their work and time (Conclusion). " [2]

In the above paragraph, the italic words in brackets are the structure analysis in the PPT courseware. Is this analysis right? Let's first answer this question: what on earth is "deductive reasoning" or "deduction"? "Deduction" is a term in logic category. In logic, reasoning is usually divided into deduction, induction and analogy. Deduction means "a process of reasoning using general rules or principles to form a judgment about a particular fact or situation." ${ }^{[3]}$ The most common form of deduction is categorical syllogism. Syllogism is a reasoning method of getting a judgment or conclusion from other two judgments.

For example, Mao Zedong (ole-fashioned spelling: Mao Tsetung) said in his essay Serve the People: "To die for the people is weightier than Mount Tai, but to work for the fascists and die for the exploiters and oppressors is lighter than a feather. Comrade Zhang Side (ole-fashioned spelling: Chang Szu-teh) died for the people, and his death is indeed weightier than Mount Tai." [4]

Here, Mao Zedong's words contain a typical deduction of syllogism. The first judgment (also called major premise) is "To die for the people is weightier than Mount Tai". The second judgment (also called minor premise) is "Comrade Chang Szu-teh died for the people". And the third judgment (also called conclusion) is "his death is indeed weightier than Mount Tai".

The above mentioned Paragraph 4 analyzed in the PPT courseware is obviously not the case because there is neither a major premise nor a minor premise in it, let alone the conclusion derived from it. In fact, the first sentence of the paragraph is a statement. It tells us "not get behind". The following sentences explained why we shouldn't get behind. They are "reasons". Therefore, this kind of method or structure should be called "a statement supported by some reasons".

Of course, in broader sense, deductive reasoning does not only refer to syllogism, it also concludes immediate inference, relational inference, association inference, disjunctive inference, hypothetical inference, dilemma and modal inference. ${ }^{[5]}$ All these inferences are based on the principle of deriving a new judgment or conclusion from one or more already known judgments. The paragraph structure analysis in the PPT courseware is obviously not the case of any of the deductive reasoning forms because it does not derive a new judgment from one or more old ones. Hence, it is not deduction at all.

\subsection{Induction or topic supported by some reasons /examples?}

Now let's examine another paragraph analysis in the PPT courseware, Paragraph 8 of Section A in Unit One, Book One of New Horizon College English Reading and Writing (Second Edition):

"(Induction) Learning a foreign language has been a most trying experience for me, but one that I wouldn't trade for anything (As an echo of Para. 1). Not only did learning another language teach me the value of hard work, but it also gave me insights into another culture, and my mind was opened to new ways of seeing things. The most wonderful result of having learned a foreign language was that I could communicate with many more people than before. Talking with people is one of my favorite activities, so being able to speak a new language lets me meet new people, participate in conversations, and form new, unforgettable friendships (Concrete reasoning). Now that I speak a foreign language, instead of staring into space when English is being spoken, I can participate and make friends. I am able to reach out to others and bridge the gap between my language and culture and theirs (Conclusion)." [2]

In the above paragraph, the italic words in brackets are the structure analysis in the PPT courseware. Is this analysis right? We should also make it clear what "induction" is. Induction, just as deduction, is a term in logic category. Induction means "a process of reasoning using known facts to produce general rules or principles." [3] Induction is a form of reasoning which derives a general conclusion from the analysis of some special cases. It consists of two parts: the premise and the conclusion. The premise are some known facts, some individual or specific judgments or statements. The conclusion is a supposition or a general statement derived from the premise through reasoning.

For example, "the angle sum of a right angle triangle is $180^{\circ}$; the angle sum of an acute triangle is $180^{\circ}$; the angle sum of an obtuse triangle is $180^{\circ}$. Since the right angle triangle, the acute triangle and the obtuse triangle encompass all of the triangles, therefore the angle sum of any triangle is $180^{\circ}$. This analysis derives a general conclusion that the angle sum of any triangle's interior angles is $180^{\circ}$ from three individual known facts that the angle sum of a right angle triangle is $180^{\circ}$, the angle sum of an acute triangle is $180^{\circ}$, and the angle sum of an obtuse triangle is $180^{\circ}$. Such mold of reasoning is called induction." [6]

Moreover, induction can be divided into two categories: perfect induction and imperfect induction. The premise of perfect induction considers all of the components in a category and its conclusion does not exceed the scope of the premise judgment, thus the conclusion must be true; while, the premise of imperfect induction only considers parts of the components in a category and the conclusion makes a general judgment which exceeds the scope of the premise judgment, thus the conclusion may not be true. For example, "Since gold, silver, copper and iron are conductive, and subsequently they are all metals, therefore, all metals are conductive." [5] This is an imperfect induction, and its conclusion may not be true because the general judgment in the conclusion exceeds the scope in the premise.

The above mentioned Paragraph 8 , analyzed in the PPT courseware, is obviously not the case above because there are neither several known facts or specific judgments as a premise, nor a supposition or a general judgment derived from the premise through reasoning as a conclusion. In the first sentence of Paragraph 8 , the first half sentence acts as a link to the previous content while the second half carries forward the new topic of this paragraph. The following sentences explain 
why "learning a foreign language has been a most trying experience for me, but one that I wouldn't trade for anything". These sentences tell us the reasons or causes. Therefore, this method or device of paragraph writing should be called "a topic supported by some reasons or causes", occasionally it can also be called "a topic supported by some examples".

Such fallacies occur in many units of the PPT courseware. Due to limited space, it is not necessary to expound them all in this paper.

\section{Root Causes of the Problems in E-Resources}

In the above section, with two examples, the author expounds the deficiencies of the electronic resources in present China by demonstrating the fallacies in the college English PPT courseware. The author analyzes the root causes to be the following aspects:

1) Inadequate attention. The written materials of New Horizon College English Reading and Writing were comprised by around a hundred of English specialists from numerous universities in the country, yet the preparation and production of the matching courseware was only delegated to one university_-Air Force University Of Engineering.

2) Lack of manpower, material resources and time. The ability of one school's faculty is very limited. The faculty in charge of producing the courseware must have individuals not only with high levels of English proficiency, but also highly skilled with PPT. However, due to their demanding job, they may not have enough time to attentively and carefully edit the material.

3) Influence of the lack of knowledge in reasoning. The 1987 edition of the high school Chinese language textbooks introduced 8 argumentative reasoning methods; but the 1990 edition of the above textbooks deleted deductive reasoning, inductive reasoning and the method of analogy. [7] This deletion led to a common confusion of a resemblance between the citation and deduction method, as well as the melting pot of deductive reasoning, inductive reasoning, and citation or exemplification.

4) Cultural interference in language transfer. From the reasons stated above, it is evident that the Chinese concept of "deduction" and "induction" is much less distinct / concrete (or covers a broader range) than their English counterparts. For instance, some people define the process of "inductive reasoning" as stating the facts first, explaining the rationale, providing the reasons and at last coming to the conclusion; and define the process of "deductive reasoning" as making a thesis and then providing evidence to further explain their reasoning ${ }^{[8]}$. Yet, in Chinese language education, some other people define the process of "deductive reasoning" as teaching the rules of grammar first then letting students practice using these rules; and define the process of "inductive reasoning" as students in the learning process, through imitation, repetition and other activities summing up the grammar rules ${ }^{[9]}$. Applying Chinese broader definition of reasoning onto the more concrete English counterpart will inevitably generate fallacies in the target language, thus, creating much confusion.

\section{Conclusions and Recommendations}

From the flaws and shortcomings of our progressive e-education as discussed above, we can see that the quality of some electronic resources is very worrisome. Especially when such electronic resources are widespread among the entire nation's teachers and students alike, if the quality of the electronic content is not improved, it will cause havoc, spread false information and will eventually confuse the entirety of the population. Therefore, with the current speed of technological advancement, the relevant departments should highly value the quality of electronic resources, strengthen management, increase manpower, and expand material resources. Thus, production of electronic teaching materials should be on par with textbook materials, vigilant, heedful and strive to eliminate erroneous views or controversial statements. Only through this, can there be a positive development of e-education.

\section{References}

[1] Shutang Zheng, New Horizon College English Reading and Writing (Second Edition), Beijing: Foreign Language Teaching and Research Press, 2008

[2] Caixia Chen, "New Horizon College English", [DB/OL], Beijing: Air Force University of Engineering, Foreign Language Teaching \& Research

Press,http://old.heep.cn/teachingresource.php?class_where $=1 \&$ where $=83$ 7, 2007.

[3] D. Summers and Yuan Zhu, Longman Dictionary of English language \& Culture (English-Chinese), Beijing: The Commercial Press, 2004, PP. 449 \& 888.

[4] Tsetung. Mao, "Serve the People", Serve the People / In Memory of Norman Bethune / The Foolish Ole Man Who Removed the Mountains, Beijing: The Commercial Press, 1972, p. 3.

[5] Qingxiang Bai, Shumei Wei and Jiafang Gao, The logic Basis, Beijing: Chinese People's Public Security University Press, 2003, pp.107-167, 168-179.

[6] Brent E. Turvey and Meijin Li, et al, Offender Profiling —_Introduction to Behavioral Evidence Analysis, Beijing: Chinese People's Public Security University Press, 2005.

[7] Linsen Chen and Ziyun Wan, "Necessary Knowledge of Deduction and Induction", Journal of Jiujiang Teachers' College (Philosophy and Social Sciences Edition), no.2, PP. 95-96, April 1996.

[8] Jigang Cai, "Influence of CET Writing Requirements and Scoring Criteria on Chinese Students' Compositions", Journal of PLA University of Foreign Languages, vol.25, no.5, September 2002.

[9] $\mathrm{Mi} \mathrm{Li}$, "Use of Deduction and Induction in Non-English Major Teaching", Journal of Chengdu University (Social Science), no. 4, pp.80-81, October 2001. 